\title{
Ambiguity in argument
}

\author{
Jan Albert van Laar* \\ Department of Theoretical Philosophy, University of Groningen, Groningen 9712 GL, The Netherlands
}

(Received 8 September 2009; final version received 11 February 2010)

\begin{abstract}
The use of ambiguous expressions in argumentative dialogues can lead to misunderstanding and equivocation. Such ambiguities are here called active ambiguities. However, even a normative model of persuasion dialogue ought not to ban active ambiguities altogether, one reason being that it is not always possible to determine beforehand which expressions will prove to be actively ambiguous. Thus, it is proposed that argumentative norms should enable each participant to put forward ambiguity criticisms as well as self-critical ambiguity corrections, inducing them to improve their language if necessary. In order to discourage them from nitpicking and from arriving at excessively high levels of precision, the parties are also provided with devices with which to examine whether the ambiguity corrections or ambiguity criticisms have been appropriate. A formal dialectical system is proposed, in the Hamblin style, that satisfies these and some other philosophical desiderata.
\end{abstract}

Keywords: active ambiguity; argument; critical discussion; equivocation; persuasion dialogue; pseudo-agreement; pseudo-disagreement

\section{Introduction}

Argumentative types of dialogue can be hampered by expressions that are ambiguous or equivocal. A participant can show dissatisfaction with such ambiguities by disambiguating the formulations he has used or by inciting the other side to improve upon their formulations. A typical example can be found in the case where W.B. had been arrested both for drink and driving and for driving under suspension. In an attempt to move his car to a car park without breaking the law, 'W.B. pushed the car, walking next to it, while he operated the steering wheel through the open window of the left car door' (Dutch jurisdiction: HR, 12 June 1990, NJ 1991, 29). W.B., however, disagreed with the police officer that his behaviour constituted driving (in Dutch: besturen, a word derived from the Dutch word for steering). In the officer's understanding of the term, W.B. had driven a car, while in the way W.B. himself understood it, he had not. Expressions that are ambiguous in ways that hinder argumentative discussion by inciting misunderstanding (Naess 1953, 1966; van Eemeren and Grootendorst 1992, 2004) or by masking argumentative weaknesses (cf., on equivocation, Mackenzie 1988, 1990; Walton 1996) will be referred to in this paper as actively ambiguous. Two issues will be examined. What notion of active ambiguity is appropriate for a normative model of argument? and In what ways should discussants themselves deal with issues of active ambiguity?

To answer these questions, I will develop a normative model of persuasion dialogue (cf. Walton and Krabbe 1995) or critical discussion (van Eemeren and Grootendorst 2004), extended by devices with which to solve ambiguity problems. In a persuasion dialogue, two parties start from a difference of opinion and attempt to resolve this difference on, what they perceive to be the merits of the case, partly by trying to persuade each other and thereby presenting the pros and cons of the issue.

Walton and Krabbe (1995) distinguish six main types of dialogue: persuasion dialogue, negotiation dialogue, deliberation dialogue, inquiry dialogue, eristic dialogue and information

*Email: j.a.van.laar@rug.nl 
seeking dialogue. This typology has been widely applied in artificial intelligence (Parsons, Wooldridge, and Amgoud 2003; McBurney and Parsons 2002; Prakken 2005; Wells and Reed 2005). For each type of dialogue, a distinction can be made between a descriptive and a normative approach (Krabbe and van Laar 2007). Given a main goal that in part characterises a type of dialogue, norms can be stated that, in a theorist's eyes, are to be followed in order for the participants to achieve this main goal. Alternatively, a theorist can use empirical means in order to arrive at an adequate description of a dialogue type, now considered as a cultural artefact referred to by van Eemeren and Houtlosser (2005) as argumentative activity type (p. 76), including a description of the conventions and rules that the participants in that dialogue type happen to impose upon each other. The question arises: if an argument is put forward in a dialogue of type $\mathrm{D}$, should we then evaluate this argument with the norms that further the main goal of that type of dialogue? In contrast to Walton (1998), who answers affirmatively (p. 30), I start from the assumption that if one is interested in the argumentative merits of an argument, it can be evaluated with the appropriate norms of persuasion dialogue, regardless of the type of dialogue in which an argument is situated. The reason is that the norms of persuasion dialogue, unlike those of the other dialogue types, explicate the pretences with which argumentative moves are brought forward (in Section 2, I will elaborate on these pretences). ${ }^{1}$ This paper is concerned with norms for persuasion dialogue that enable the participants to deal with active ambiguities. These norms are meant to apply to argumentative contributions, whatever their specific dialogical setting.

The result is a formal dialectical dialogue system with which the moves available to an agent at each stage can be univocally determined. Specific to the model developed in this paper is that the agents are enabled to raise the issue of active ambiguity in various ways depending on the circumstances, and deal with such procedural moves in a critical manner. In this way, the paper develops Hamblin's (1970) programme for a theory of charges or procedural objections (chap. 8) that is immanently dialectical (Krabbe 1997) in providing the agents with the dialogical means to address the argumentative problems and fallacies they encounter. The resulting system, ambiguity dialectic, constitutes a proposal for dealing with active ambiguities in argumentatively reasonable ways. First, the model can be used for the analysis and evaluation of actively ambiguous arguments and concessions as well as the moves with which participants themselves attempt to solve ambiguity problems. Second, the model can be used to enrich argumentative interaction protocols by enabling the agents to address active ambiguities.

In Ambiguity Dialectic (specified in Section 5), four distinct components for dealing with active ambiguity are integrated into a model of persuasion dialogue: (1) a component that enables a protagonist (or proponent) of a thesis or standpoint to offer a self-critical ambiguity correction, (2) a component that enables the protagonist to raise an ambiguity criticism against his antagonist (or opponent), (3) a component that enables the antagonist to offer a self-critical ambiguity correction and (4) a component with which the antagonist can offer an ambiguity criticism against the protagonist. $^{2}$

Section 2 will first introduce the dialogical approach to argument and criticism. Section 3 will provide an explication of the term active ambiguity. Section 4 will introduce a number of philosophical desiderata of a dialogue system that can accommodate active ambiguities, while Section 5 will outline a dialogue system along these lines and a number of examples that illustrate the system.

\section{Arguments and criticisms in ambiguity dialogues}

The dialogue system ambiguity dialectic is a close relative of the model of critical discussion developed by van Eemeren and Grootendorst (2004), and the family of models of persuasion 
dialogue developed by Walton and Krabbe (1995) and adapted for the purposes of artificial intelligence by, for example, Prakken (2005), McBurney and Parsons (2002), Parsons et al. (2003) and Wells and Reed (2005). In line with these dialectical approaches, the notions of 'argument' and 'criticism' are here understood from the perspective of a critical exchange between a protagonist and an antagonist. The protagonist defends a standpoint against the criticisms of an antagonist using propositions that the antagonist has committed to at the start of the exchange.

An ambiguity dialogue, that is, a dialogue according to the rules of ambiguity dialectic, starts with a situation in which the protagonist and the antagonist are assumed to have a difference of opinion that they intend to resolve on what they perceive to be the merits of the case. It suffices to say that the parties are assumed to disagree, for it is not to be excluded that the parties come to decide at a later stage that the assumed difference of opinion was merely verbal rather than substantial.

At a preliminary stage, the antagonist has committed to a possibly empty set of formulated propositions called her initial concessions (cf. Barth and Krabbe 1982). The protagonist's first move in the dialogue expresses a standpoint. The individual aim of the protagonist is to demonstrate to the antagonist that the initial concessions that the latter has made also commit her to the acceptability of the former's intended standpoint. ${ }^{3}$ The individual aim of the antagonist is to demonstrate to the protagonist that it is possible to maintain a critical stance vis-à-vis the standpoint, notwithstanding the initial concessions. This dialectical division of labour (Rescher 1977 ) is instrumental for the realisation of the main goal of the dialogue, which is the resolution of the difference of opinion. ${ }^{4}$ Because the individual aim of the protagonist is to show that the initial commitments of the antagonist also commit her to the standpoint, the protagonist needs to bridge the gap between the initial concessions and that standpoint by reasoning, stepwise, from the concessions to the standpoint. Thus, within this framework, an argument will only be successful through the use of ex concessis argumentation. For the protagonist's strategy to be successful against an antagonist with some acumen, both the reasons and the connection premises within this configuration of arguments must either be conceded or they must follow from what the antagonist concedes to be acceptable logical procedures.

What resolution amounts to is specified by the rules that constitute an ambiguity dialogue. The general idea is that the participants have resolved their difference of opinion if either the protagonist has given up defending the standpoint or if the antagonist has given up challenging the protagonist's defence after having had all the opportunities they themselves considered necessary to achieve their individual persuasion aims. The discussion rules provide an explication of what it means to work towards a resolution of a difference of opinion. Given that we consider some differences of opinion unresolvable - for example, because we lack the information with which to decide the issue or because the disagreement is overly intractable, and given that in a reasonable persuasion dialogue the participants cannot be forced to prematurely terminate their persuasion dialogue, the model should enable the participants to converse without ever making a decision to terminate the conversation. For practical purposes, additional rules can be adopted that do guarantee a determinate outcome in favour of one of the participants.

In an ambiguity dialogue, participants converse at two levels (cf. Krabbe (2003) on meta-level dialogue; cf. McBurney and Parsons (2002) on the control layer of a dialogue, pp. 323-325). At a ground level, the parties exchange arguments and criticisms. The antagonist is allowed to pose critical questions, which must be understood as requests for argument. These critical questions enable the antagonist to force the protagonist to accomplish the burden of proof, without having to take on a burden of proof herself, as in the case of attempts at defeating the protagonist's reasoning using a rebutting argument, an undercutting argument or a premise attack (cf. Pollock 1995, pp. $40-41$; cf. Prakken 2005, p. 1013). In response to a challenge 'why $\varphi$ ?', the protagonist has a prima facie obligation to offer an argument ' $\psi$ so $\varphi$ ', such that $\psi$ is the argument's reason and $\psi \rightarrow \varphi$ is the argument's connection premise. As challenges always target elements that are part of an 
already presented argument, the set of arguments that have been put forward at a particular stage in the dialogue form a tree-shaped configuration called the global argument of that stage, constituted by various local arguments presented at previous stages. At earlier stages, the global argument will consist of only one local argument. Also the initial standpoint, at any stage at which a single reason has not yet been put forward, will be dealt with as the global argument at that stage, having zero premises. At a meta-level, the parties can deal with the adequacy of the choice of words as well as with the appropriateness of ambiguity criticisms or of self-critical, spontaneous disambiguations.

In the context of an ambiguity dialogue, the term 'argument' refers to pieces of reasoning, used for persuasive purposes, ${ }^{5}$ that are reasonable in the sense of producible by the rules of ambiguity dialectic. This notion can be used to define 'argument' in a broader sense, including arguments that violate discussion norms, in the following way. Presenting an argument, in this broader sense, is to offer a verbal contribution to a dialogue (which can be of any type) such that the arguer pretends that the addressee will be capable of reconstructing a flawless ambiguity dialogue from the text. In this reconstructed ambiguity dialogue, the arguer plays the role of the protagonist and the addressee the role of antagonist, whereby the antagonist poses the critical questions she considers pertinent but is nonetheless persuaded by the protagonist's defence and ultimately gives up her critical stance towards the protagonist's standpoint (cf. Krabbe's (2001) dialogical definition of the notion of 'an argument that can reasonably convince someone'). The presentation of an argument, in the broader sense, can thus be seen as the expression of three pretences, all of them explicable in terms of the normative model of a persuasion dialogue: a pretence to the argument having a clear enough dialogical structure, a pretence to the argument being reasonable according to a set of argumentative norms and a pretence to the argument being effective by leading the antagonist to withdraw her critical stance towards the standpoint. ${ }^{6}$ A similar story can be told about a more inclusive notion of 'criticism'. In what follows, I will use 'argument' in the stricter, more exclusive sense of a contribution to an ambiguity dialogue.

At each stage, a participant has a commitment store that contains the propositions that he or she is committed to at that stage of the dialogue (Hamblin 1970; Walton and Krabbe 1995). In ambiguity dialectic, the protagonist's commitment store remains a singleton that contains the global argument at that stage. This commitment of the protagonist, however, can become increasingly complex due to the addition of arguments and subsequent disambiguations. The antagonist's commitment store contains the concessions which can change through subsequent disambiguations and the number of concessions can increase through the antagonist accepting various readings of one and the same concession.

\section{Active ambiguity}

What is the pejorative sense of ambiguity as this term is used in an argumentative context? When discussing a definition, I will prepare for an appropriate division of labour between the protagonist and the antagonist by pointing out who is likely to profit from and who is likely to pay for the ambiguity left unnoticed and unresolved. The latter party will be given the meta-linguistic devices with which to bring the discussion back on track.

The following argument can be used as part of a case in favour of the contention that the English term 'ambiguous' can be used ambiguously, in its pejorative sense, on some occasions. One might, on a superficial reading of it, consider the argument as having two acceptable reasons and an acceptable connection premise that is left implicit, but a clearly unacceptable standpoint:

(1) Almost all English expressions are ambiguous. (2) If a speaker uses an expression that is ambiguous, then we can object to the use of that expression. Therefore, (3) we can object to the use of almost all English expressions. 
I am interested here in the sense of the term 'ambiguous' that makes reason (2) acceptable while making reason (1) quite implausible. 'Ambiguity' in this pejorative sense and when applying to argumentative contexts will be called 'active ambiguity'. The definition has three clauses and is restricted to the propositional content of speech acts. Thus, I will refrain from dealing with ambiguities in illocutionary force and with ambiguities in the super-sententional structures of a textual contribution, such as with respect to whether something is an argument or an explanation, or to whether reasons are to be seen as linked (or compound) or convergent (or multiple), and so forth.

First, for an expression to be actively ambiguous in a particular dialogue, it must allow of various readings in that dialogue, even after having taken the contextual clues into account. For example, particular uses of the word 'bank' can be contextually ambiguous: 'I've seen John walking to the bank therefore his leg must have healed.' Also, expressions that are vague, in the sense of admitting of borderline cases, having fuzzy edges and enabling the construction of sorites arguments (Keefe 2000, pp. 6-7), typically allow various readings within specific circumstances and are consequently candidates for active ambiguities. Contextual ambiguity has been studied extensively in 'word sense disambiguation' in computational linguistics (see for overviews, Ide and Veronis 1998; Navigli 2009). Active ambiguity applies to expressions that are used in a particular situation and that in this situation allow various readings even when having taken the contextual clues into account.

Second, the ambiguity is not overt. It is not made clear to the addressee that the expression admits of more than one reading, for example by conveying the message that the expression is to be understood in terms of both readings. Active ambiguity is not a figure of style. In his introduction to Thoreau's his pamphlet 'On the duty of civil disobedience' Sharp explains that Thoreau used the term 'civil' in 'civil disobedience', overtly in two distinct senses, referring to politeness and humaneness as well as to what befits us as members of a community of citizens (Thoreau 1963/1849, p. 3). The above bank example can easily be imagined to contain a covertly ambiguous occurrence of 'bank'.

Third, among the covert and contextual ambiguities, a further distinction can be made. Some can be expected to allow interpretational options, such that choosing one over the other has consequences for whether or not the standpoint, reason or connection premise is acceptable to the antagonist. Other interpretational divergences, however, are so overly subtle, fine-grained, far-fetched or irrelevant to the topic at hand that choosing one reading over the other would be inconsequential to the course of the dialogue. The first is what constitutes an active ambiguity.

If the parties are only interested in John's physical well-being, the sentence can be covertly ambiguous without leading to any interactional problems. Of course, the interlocutor may desire to know what the speaker has in mind when using the term 'bank', but for the argumentative purpose of this dialogue, a request for disambiguation would probably initiate an irrelevant detour. Similarly, a request for more precision seems inappropriate when a protagonist states that Mozart was a musical child, for it is clear that he was musical in all relevant senses (cf. Pinkal (1995) for a supervaluationist theory of reasoning that elaborates this idea). Again, the interlocutor can be interested in what the speaker more specifically has in mind, but this interest extends beyond the aim of resolving disputes. Active ambiguity is not merely a communicative but also an interactional phenomenon.

Nevertheless, some covertly ambiguous expressions are plausible candidates for creating a confusion that can influence the course of the verbal interaction and are consequently actively ambiguous. Think of the term 'unbearable suffering', which constitutes a ground for exemption from liability under Dutch euthanasia law. In a much discussed case, a family doctor had provided the former Dutch senator Brongersma with a lethal potion. Brongersma had been weary of life (in Dutch: levensmoe) and according to the doctor's defence, Brongersma had complied with the criterion of unbearable suffering. Thus, in this context, the vague expression unbearable suffering 
triggers two kinds of readings - does it include mental disorders such as extreme depression or does it not? - and becomes actively ambiguous (van Laar 2003, chap. 8).

Or take the driving example from the introduction. Suppose the police officer stated to W.B.:

Officer: You're driving a car while your license is suspended therefore a fine is in order.

Then, W.B., were he sufficiently reflective, would note two senses in which to drive can be taken in this dialogue situation. Either the term is taken in a stricter sense, excluding W.B.'s actions, or in a broader sense, including them. If W.B. detected these interpretational options, he would subsequently notice that accepting the officer's reason in the broader sense need not harm his position, for in that reading the connection premise is plausibly false, while accepting it in the stricter sense would amount to losing the discussion. W.B. now has two options that might turn out opportune for him.

First, he might raise the issue of ambiguity, pointing out the two meanings that to drive admits of in this situation and the fact that he is willing to concede the officer's reason in the broader reading of the term but not in the stricter. He might want to add that his acceptance of the reason in its broader reading does not provide the officer, in his capacity as the protagonist, with a winning argumentative strategy, the reason being that W.B. does not accept the connecting premise according to which driving in this broad sense would amount to a transgression of the law.

If one party introduces an expression and the other party thinks it opportune to point out its active ambiguity, we can best refer to the raising of this issue as an ambiguity criticism. The reason is that the party who introduces an expression remains at least partly responsible for the problems to which the expression gives rise. ${ }^{7}$ Raising the issue of ambiguity amounts to the message that the other side has transgressed a norm of reasonable discussion and in doing so has harmed their own position. Therefore an ambiguity criticism can best be seen as a special kind of fallacy criticism (cf. Krabbe 2002). In the example, the antagonist (W.B.) puts forward an ambiguity criticism, given that the protagonist (the officer) is responsible for introducing the term 'to drive' in this dialogue. Note that an ambiguity criticism can be correct even if the perpetrator of the fallacy is not aware of having transgressed any norm. See the first set of examples in Section 5.7 for an illustration of how the dialogue system handles such interactions.

Second, W.B. might choose not to raise the issue of ambiguity and simply challenge the officer's reason that he had been driving a car:

W.B.: Why would you say I was driving the car?

Compared with simply challenging a reason that contains an actively ambiguous expression, raising an ambiguity criticism is, somewhat surprisingly, a more cooperative form of critique. This is because it provides the protagonist with much more information concerning how to adapt an argument in order for it to convince the antagonist.

Be that as it may, now it is the officer's turn, and the officer might note the two interpretational options. The officer might raise the ambiguity issue, pointing out that W.B. was probably challenging his reason while taking 'driving' in its strict sense, whereas it was meant in its broader sense, which the officer surmises as acceptable to W.B. The officer might add that if, as expected, W.B. accepts the broader sense of the statement, a strong persuasive strategy is available, as the officer also expects that it will be possible to convince W.B. of the proposition that 'driving' in the broader sense constitutes a violation of the law. (The disagreement appears to be substantial with respect to the connection premise, but not with respect to the reason.)

If one party first introduces an expression and this same party thinks it opportune to point out that this expression is actively ambiguous, this raising of the issue of ambiguity can best be seen as a self-critical move called an ambiguity correction. Note that an ambiguity correction can be legitimate even when one has willingly introduced an actively ambiguous expression. Raising an 
ambiguity correction amounts to admitting that an argumentative move has transgressed a norm that harmed one's own position. An ambiguity correction can best be seen as acknowledging a strategically weak move, or even a blunder, that is subsequently repaired and corrected. In the current scenario of the example, the protagonist (the police officer) can be understood as offering such an ambiguity correction.

In the first scenario, where the antagonist (W.B.) raises an ambiguity criticism, the antagonist appeals to the possibility of having accepted a reason, while the protagonist intended to express a different reading, and as a matter of fact a reading that is not acceptable to her. Hence, the antagonist appeals to the possibility of a particular kind of misunderstanding, which Naess (1966) has called pseudo-agreement. More precisely, the antagonist appeals to the feasibility of performing a speech act, that is, verbally accepting a reason, which could better have been avoided. Clearly, such a pseudo-agreement, if materialised in the speech acts of the parties, is detrimental to the antagonist's chances of winning the discussion. Pseudo-agreement is always prima facie disadvantageous to the antagonist and advantageous to the protagonist.

The protagonist's (the officer's) ambiguity correction, in the second scenario, appeals to the possibility that the antagonist challenges a reason that the protagonist intended in a different sense, and as a matter of fact in a sense that is acceptable to the antagonist. Hence, the protagonist appeals to the possibility of a different kind of misunderstanding, which Naess called pseudo-disagreement. More precisely, the protagonist appeals to the feasibility of the antagonist's performing a speech act, that is challenging the reason, that is not really appropriate and in fact undesirable from the perspective of the protagonist's individual aim. Clearly, a pseudo-disagreement, if materialised in the speech acts of the parties, is always prima facie disadvantageous to the protagonist and advantageous to the antagonist.

The kind of reasoning that is normally discussed under the heading of 'equivocation' (Mackenzie 1988; Walton 1996) must, within this argumentative setting, be seen as a special case of trading on an actively ambiguous expression that leads to or might lead to pseudo-agreements. An equivocation is an argument that contains a covertly and contextually ambiguous expression that admits of more than one reading, such that: (1) there is a reading that makes all reasons acceptable to the addressee, and (2) there is a reading that makes the connection premise acceptable, but (3) there is no reading that makes all reasons and the connection premise acceptable. These readings, for example of the connection premise, can be mixed disambiguations (Lewis 1982) where the expression at hand obtains distinct disambiguations at distinct occurrences within a sentence. This analysis of equivocation applies to the argument that trades on the term 'ambiguous' discussed at the start of this section, and also to the police officer's argument as expressed vis-à-vis W.B. An equivocation can be understood as involving more than one pseudo-agreement. Failing to notice the ambiguity and naively accepting the reasons and connection premise (due to their having an air of acceptability), leads to a complex kind of pseudo-agreement: for at least one reason as well as for the connection premise it holds that there is a reading that makes it acceptable as well as a reading that makes it unacceptable to the addressee. ${ }^{8}$

An expression, as used in a particular argumentative discussion, is only to be called an active ambiguity if its use is likely to have one of these interactional consequences rather than merely communicative effects. The ambiguity is actively problematic rather than latently so, as in the case of merely contextual ambiguities. Thus, if an expression motivates one of the participants to ponder the intended meanings, without its being the case that one interpretational option would make the assertion acceptable while the other would make it questionable, then the expression, in this context, is not actively ambiguous. However, if it can be shown that it is plausible that a pseudodisagreement, pseudo-agreement or an equivocation is at play, then it is actively ambiguous. The proper definition of 'active ambiguity', that is, of 'ambiguity' in its pejorative sense as used in argumentative situations, is that of a covert, contextual ambiguity that is of interactional consequence. 
Given that the interactional consequences are always detrimental to one of the participants, active ambiguity is also of strategic significance.

In this section, three distinctions have been drawn that will be used in the specification of ambiguity dialectic. (1) Some covert contextual ambiguities are not interactionally relevant in a discussion while others are. (2) Active ambiguities are either connected to pseudo-agreement, including the ambiguities in equivocal reasoning, or to pseudo-disagreement. In the first case, it is up to the antagonist to solve the ambiguity problem, in the second case, it is up to the protagonist. (3) Either the protagonist first introduces the expression that turns out to be actively ambiguous, or the antagonist does so. In the first case, raising the issue of ambiguity amounts to an ambiguity correction if this is done by the protagonist and to an ambiguity criticism if done by the antagonist. In the second case, raising the issue of ambiguity counts as an ambiguity criticism if this is done by the protagonist and as an ambiguity correction if done by the antagonist.

\section{Philosophical requirements of a dialogue system for ambiguity dialogues}

In Section 5, a dialectical system will be developed that satisfies the following requirements: the system should be immanently dialectical, it should strike a balance between normative bite and a tolerance of imperfections, it should implement the correct norms regarding the use of actively ambiguous expressions, it should be organised in accordance with a dialectical division of labour, it should strike a balance between enabling meta-remarks and addressing the topic at hand, and it should make use of a clear conception of disambiguation. I will discuss these six desiderata in turn.

(1) A theory of argumentation is meant to assist agents who are dealing with a contentious issue. The disagreement can easily extend to the criteria with which to decide whether expressions are actively ambiguous. Therefore, the theory should accommodate situations where the participants disagree about whether an expression is actively ambiguous. In Krabbe's words, the theory is to be 'immanently dialectical', in the sense of providing the participants with the means to solve their own problems, ${ }^{9}$ without requiring recourse to alleged objective criteria and without the presence of a third party who acts as a judge who has the power to settle disagreements, rather than resolve them. ${ }^{10}$ Consequently, the dialogue system to be introduced will contain the option to perform speech acts with which ambiguous arguments or ambiguous concessions can be disambiguated, but it will also contain the speech acts with which the linguistic admissibility of a disambiguation can be challenged and with which the interactional relevance of an ambiguity correction or ambiguity criticism can be tested. ${ }^{11}$

(2) The theory is to provide norms at two distinct levels. First, the theory must make it clear what a commitment to the clear use of language amounts to, as far as the avoidance of ambiguities is concerned. The ideal is simply to steer clear of active ambiguities. However, there are a number of reasons not to adopt this norm as constitutive of reasonable persuasion dialogue. ${ }^{12}$ First, a certain degree of opportunism when attempting to achieve one's individual persuasive aim is instrumental to genuine dispute resolution. Therefore we should be careful to exclude opportunistic choice of word, even if a participant chooses terms that allow of more than one reading within the context of utterance. Second, a participant might be in a position where it remains unclear to him or her whether an expression is actively ambiguous or not. For we cannot expect a participant to be capable of calculating all linguistically admissible readings of each utterance and we cannot expect that a participant, at the moment he wants to put the expression to use, has access to the information with which to decide what readings of a sentence will turn out acceptable or unacceptable to the other side. Thus it is useful to have a set of norms that tell us how to respond to alleged active ambiguities in a way that facilitates the resolution of our differences of opinion. Consequently, the 
desired dialogue model must provide rules on the one hand and allow for rule-violations on the other hand. These requirements can be balanced by distinguishing between two kinds of norms.

There is a regulative rule that prohibits active ambiguities and this regulative rule is implemented by constitutive rules that enable the participants to deal with violations of this ideal in the best possible manner. In this way, a stricter model of persuasion dialogue, which precludes the parties from employing actively ambiguous expressions, is embedded in a looser model of persuasion dialogue that does not make it impossible for the parties to use such expressions (intentionally or by accident) but instead, enables them to raise the issue of ambiguity and to improve upon their language if they consider it necessary (cf. Mackenzie $(1988,1990)$ for a similar solution to the problem of modelling rule violations.) Consequently, by adopting a model that strikes a balance between normative bite and tolerance of imperfections, it is possible to commit oneself to the ideal of a language that is free of active ambiguities, while adopting an appropriate measure of realism, leniency and flexibility.

(3) In addition, the model must implement the correct norm. First, given the explication of the pejorative kind of ambiguity as active ambiguity, provided in Section 3, any multiplicity in meaning that is either not contextual, not covert or not interactionally relevant should not be banned as actively ambiguous from argumentative discussion. Thus, parties should not be discouraged from using expressions that are merely contextually ambiguous. In order to keep things simple, it will be assumed that all interactionally relevant ambiguities are covert ambiguities. In other words, we will not be dealing with literary uses of contextual ambiguity. Second, the consequences of having used an actively ambiguous expression should not be overly severe. For example, if the antagonist successfully points out an active ambiguity and forces the protagonist to disambiguate the argument, it remains entirely up to the protagonist to make the disambiguation choices that he considers appropriate and opportune. ${ }^{13}$ The protagonist retains the right to choose how his global argument is to be understood and the antagonist how her concessions are to be taken. ${ }^{14}$ Third, a participant must be capable of choosing a mixed disambiguation whereby various occurrences of an expression within a sentence obtain a distinct disambiguation: 'John went to the bank, in the financial institution sense, near the bank, in river edge sense.' Fourth, a participant must be able to concede a sentence in terms of two or more readings: 'I am willing to accept that Mozart was musical, in all senses of the word you distinguish.' Fifth, even an expression that is the result of a proper disambiguation can turn out to be actively ambiguous itself. Thus, the model should do justice to Lewis's (1982) pessimist who reckons with the possibility that the parties will never reach a level of precision that suffices. Whatever the case may be, it is a matter to be decided by the parties. The possibility that they can agree on a proper level of precision is not excluded, but in some cases they may not agree. ${ }^{15}$ In short, the theory must implement the correct norms, enabling each party to remedy presumably real mistakes and fallacies as well as allowing them to defend themselves successfully from presumably incorrect ambiguity criticisms. ${ }^{16}$

(4) A related issue is the requirement to strike a balance between enabling the parties involved to raise the meta-issue of ambiguity by correcting themselves or by criticising the other and remaining focused on their attempts to resolve the ground level dispute which started the discussion. The proper solution is that a meta-dialogue is instrumental to and embedded in the ground level discussion. Thus, the rules will enable meta-dialogues concerning alleged active ambiguities, but also incite the parties to return to the ground level topic as soon as possible. In ambiguity dialectic, the rules are such that a participant has one shot at an alleged ambiguity, while the other side has at most two shots at criticising the ambiguity correction or ambiguity criticism, and the metadialogue terminates with a retraction of the ambiguity correction or ambiguity criticism or with a so-called forced disambiguation (to be explained below). After the retraction of an ambiguity correction or ambiguity criticism and after a forced disambiguation, the participants resume the ground level dialogue. 
(5) A further requirement is that ambiguity dialogues are based upon a distribution of rights and obligations that fits the asymmetrical, dialectical division of labour (Rescher 1977, p. xiii). As we have seen, each participant can continue to work towards his individual aim, also while dealing with ambiguity problems. As explained in the last section, if participant A is responsible for introducing an expression $\alpha$, then participant A can do something about $\alpha$ 's active ambiguity with an ambiguity correction if it harms himself and participant B can do something about it with an ambiguity criticism if it harms participant $\mathrm{B}$, such that the antagonist attempts to solve or avoid pseudo-agreements, including equivocal reasoning, and the protagonist attempts to solve or avoid pseudo-disagreements. This implementation of a division of labour goes beyond Walton's (1996) theory, according to which the proper use of language is a shared responsibility (pp. 34-35). However, Walton does not provide procedural details. ${ }^{17}$

(6) Finally, the model must make use of a clear concept of disambiguation. Disambiguation can, in the kind of context we are examining, apply to the global argument at some stage (which may, initially, happen to be just the standpoint or a single argument) or to the set of initial concessions at some stage. Suppose a global argument $G_{i}$ (or a set of concessions $C_{i}$ ) has a number of occurrences of the expression $\alpha$. Then a disambiguation of $G_{i}$ (or of $C_{i}$ ), based on $\alpha$ and a set of disambiguating reformulations $\alpha_{1}, \ldots, \alpha_{n}$ of $\alpha$, results from replacing each occurrence of expression $\alpha$ in $G_{i}$ (or in $C_{i}$ ) with one of the expressions from $\alpha_{1}, \ldots, \alpha_{n}$. Different occurrences of $\alpha$ in a sentence can be replaced by different disambiguating reformulations from $\alpha_{1}, \ldots, \alpha_{n}$. A concession $\varphi[\alpha]$, that is, $\varphi$ with an occurrence of $\alpha$, can be replaced by various disambiguations $\varphi\left[\alpha_{1}\right], \ldots, \varphi\left[\alpha_{n}\right]$, in cases where the antagonist is willing to accept a number of readings of one of her concessions. There is no restraint on what counts as a disambiguating reformulation $\alpha_{i}$ of $\alpha$. The parties are free to choose them. However, if the other side is dissatisfied and suspects that $\alpha_{i}$ is not a proper disambiguating reformulation of $\alpha$, in the sense that $\alpha_{i}$ is ruled out as such by the semantic rules shared by the parties, he may initiate a linguistic test to check whether this suspicion is right. If the number of occurrences of $\alpha$ is $\mathrm{m}$ and the number of proposed disambiguating reformulations is $\mathrm{n}$, there are exactly $n^{\mathrm{m}}$ possible disambiguations of the argument and at least $n^{\mathrm{m}}$ possible disambiguations of the concessions set.

Disambiguation is related to retraction (cf. Walton and Krabbe 1995), but differs in the important respect that the person who disambiguates remains committed to a proposition expressed by the sentence replaced, even though at certain stages in the discussion what proposition that is can still be undetermined. Consequently, if statement $\varphi[\alpha]$ is disambiguated, with $\varphi\left[\alpha_{i}\right]$ the result, then it is supposed that all propositions expressed by $\varphi\left[\alpha_{i}\right]$ in this context are also expressed by $\varphi[\alpha]$, but not vice versa (cf. Naess $(1953,1966)$ on 'precization'). According to my use of terms it is wrong to say that the protagonist was first committed to all propositions expressed by $\varphi[\alpha]$ and retracts, by disambiguating $\varphi[\alpha]$ to $\varphi\left[\alpha_{i}\right]$, all propositions expressed by $\varphi[\alpha]$ but not by $\varphi\left[\alpha_{i}\right]$. We should rather say that the protagonist was committed to the formulation $\varphi[\alpha]$ and to at least one proposition expressed by $\varphi[\alpha]$, whether or not the parties know exactly what proposition that is. After the act of disambiguation, the protagonist is no longer committed to the wording of $\varphi[\alpha]$, but is still committed to at least one proposition expressed by $\varphi[\alpha]$. The protagonist becomes committed to the wording of $\varphi\left[\alpha_{i}\right]$. Thus, seen from the perspective of the formulation, disambiguation might be called a special kind of retraction. Seen from the perspective of meaning, disambiguation is not retraction at all, but a way in which it may be made more clear what exactly a propositional commitment consists of. ${ }^{18}$

In accordance with the requirement of immanent dialectics, there is no supposition as to whether, 'as a matter of fact', a real disagreement has threatened the discussion or occurred at some point in the dialogue. Instead, the parties can never be sure what the other side has in mind, or even precisely what they have in mind themselves. What the rules implement, however, is a commitment to some proposition or propositions when making a concession or when presenting 
an argument. The model, however, does assume that the parties can, at least sometimes, come to agree on disambiguation relations between sentences, so that they agree on the one sentence $\varphi\left[\alpha_{i}\right]$ being more precise than another $\varphi[\alpha]$, thereby excluding some rival disambiguations $\varphi\left[\alpha_{j}\right]$. That the proposition expressed remains hidden is what enables the parties to decide to consider (construe) some agreements and disagreements as instances of pseudo-communication and others as substantial and real.

Fulfilling these six requirements will lead to a dialogical or dialectical theory that has both normative bite and at the same time is suitable for real and imperfect reasoners in an argumentative discussion.

\section{Ambiguity dialectic}

\subsection{Definitions and conventions}

In the following, the letters $\alpha, \alpha_{1}, \alpha_{2}, \ldots, \beta, \beta_{1}, \beta_{2}, \ldots$, etc., are variables for atomic sentences of the language, while $\varphi, \psi, \ldots$, etc., are variables for sentences of the language, atomic or complex. $G_{i}$ refers to the global argument at stage $i$, that is, the tree-shaped configuration of local arguments put forward by the protagonist at the stages $j, j \leq i$. The global argument at $i$ is the unique item in the commitment store of the protagonist at that stage. $\mathrm{G}_{i}$ can contain the initial standpoint, a single argument in favour of the standpoint, a chain of arguments or a disambiguation of them. The expression $f\left(G_{i} ; \alpha ; \alpha_{1}, \ldots, \alpha_{n}\right)$ refers to a result achieved by replacing each occurrence of expression $\alpha$ in $G_{i}$ with expressions from $\alpha_{1}, \ldots, \alpha_{n}$. Different occurrences of $\alpha$ can be disambiguated in different ways. The expressions $\alpha_{1}, \ldots, \alpha_{n}$ are in this manner presented as disambiguating reformulations of $\alpha . C_{i}$ refers to the commitment store of the antagonist, consisting of her initial concessions, at stage $i . C_{i}$ can only change due to disambiguations. The term $f\left(C_{i} ; \alpha ; \alpha_{1}, \ldots, \alpha_{n}\right)$ refers to a result achieved by replacing each occurrence of expression $\alpha$ in $C_{i}$ with expressions from $\alpha_{1}, \ldots, \alpha_{n}$. Different occurrences of $\alpha$ can be disambiguated in different ways. A concession can be substituted with more than one disambiguation. The expressions $\alpha_{1}, \ldots, \alpha_{n}$ are in this manner, again, presented as disambiguating reformulations of $\alpha$. An expression $\alpha$ is disqualified at a stage $i$, if at an earlier stage $\alpha$ has been the focus of an ambiguity correction, also called a spontaneous disambiguation, or of an ambiguity criticism, and if this disambiguation or criticism has not been withdrawn in the meantime.

Ambiguity dialectic will be characterised by four kinds of rules: locution rules, commitment rules (cf. update rules in Parsons et al. 2003), dialogue rules (cf. rationality and dialogue rules in ibid; cf. combination rules in McBurney and Parsons 2002; cf. structural rules in Walton and Krabbe 2005) and win-and-loss rules (cf. termination rules in McBurney and Parsons 2002).

\subsection{Locution rules}

There are six types of speech act or locution that can only be used by the protagonist:

L1: Initial standpoint:

$\operatorname{ST} \varphi$.

L2: Local arguments:

$\psi \mathrm{SO} \varphi$

L3: Spontaneous disambiguations of arguments:

$\operatorname{SD} f\left(G_{i} ; \alpha ; \alpha_{1}, \ldots, \alpha_{n}\right)$

The protagonist can present a spontaneous disambiguation of his global argument at stage $i$, that is, an ambiguity correction, using $\alpha_{1}, \ldots, \alpha_{n}, n>1$, as disambiguating reformulations of $\alpha$. The newly disambiguated global argument, $f\left(G_{i} ; \alpha ; \alpha_{1}, \ldots, \alpha_{n}\right)$, need not contain an occurrence of each of the disambiguating reformulations in the list $\alpha_{1}, \ldots, \alpha_{n}$. However, it is 
assumed that the speaker makes it clear that his disambiguation is based upon this linguistic analysis of $\alpha$ 's ambiguity. This assumption also applies to similar locutions below.

L4: Forced disambiguations of arguments:

$\operatorname{FD} f\left(G_{i} ; \alpha ; \alpha_{1}, \ldots, \alpha_{n}\right)$

The protagonist can present a forced disambiguation of the global argument at stage $i$, using $\alpha_{1}, \ldots, \alpha_{n}, n>1$, as disambiguating reformulations of $\alpha$ offered in response to a critical move by the other side.

L5: Disambiguation withdrawals: $\quad \mathrm{WIG}_{i} ; \alpha$.

The protagonist can return to the global argument at $i$, thereby withdrawing a disambiguation $f\left(G_{j} ; \alpha ; \alpha_{1}, \ldots, \alpha_{n}\right), i<j$, and reinstalling $\alpha$ as no longer disqualified.

L6: Global arguments:

$G_{i}$

The protagonist can present his global argument at that stage.

There are five types of locution that can only be used by the antagonist:
L7: Challenges:
$\mathrm{WH} \varphi$

The antagonist can challenge a standpoint, reason or connection premise.

L8: Spontaneous disambiguation of concessions: $\quad \operatorname{SD} f\left(C_{i} ; \alpha ; \alpha_{1}, \ldots, \alpha_{n}\right)$

The antagonist can present a spontaneous disambiguation of the set of concessions at stage $i$, that is, an ambiguity correction, with $\alpha_{1}, \ldots, \alpha_{n}, n>1$, as disambiguating reformulations of $\alpha$.
L9: Forced disambiguation of concessions:
$\operatorname{FD} f\left(C_{i} ; \alpha ; \alpha_{1}, \ldots, \alpha_{n}\right)$

The antagonist can present a forced disambiguation of the set of initial concessions at stage $i$, using $\alpha_{1}, \ldots, \alpha_{n}, n>1$, as disambiguating reformulations of $\alpha$, offered in response to a critical move by the other side.

L10: Disambiguation withdrawals: $\quad \mathrm{WIC}_{i} ; \alpha$.

The antagonist can return to the set of concessions at stage $i$, thereby withdrawing a disambiguation $f\left(C_{j} ; \alpha ; \alpha_{1}, \ldots, \alpha_{n}\right), i<j$, and reinstalling $\alpha$ as no longer disqualified.
L11: Pseudo-agreement analyses:
$\mathrm{CO} \varphi\left[\alpha_{i}\right] ; \mathrm{WH} \varphi\left[\alpha_{j}\right] ?$

The antagonist can present an analysis of a pseudo-agreement by stating that she would have been willing to concede a sentence in one reading without accepting it in another reading.

There are five types of locution that can be used by the protagonist and antagonist:

L12: Ambiguity criticisms: $\quad \alpha \mathrm{AA} \alpha_{1}, \ldots, \alpha_{n}$

A participant can raise an ambiguity criticism, such that $\alpha$ is characterised as actively ambiguous between $\alpha_{1}, \ldots, \alpha_{n}, n>1$.

L13: Relevance criticisms:

RE?

A participant can challenge the relevance of an ambiguity criticism or a spontaneous disambiguation. 
L14: Linguistic criticisms:

LI?

A participant can challenge the linguistic admissibility of an ambiguity criticism or a spontaneous disambiguation.

L15: Ambiguity criticism withdrawals: $\quad$ WI $\alpha$

A participant can withdraw the criticism that $\alpha$ is actively ambiguous.

L16: Surrenders:

GIVE UP

A participant can give up.

\subsection{The commitment rules}

The commitment store of the antagonist at stage $i, C_{i}$, contains the concessions the antagonist has made, vis-à-vis the protagonist, at a preliminary stage of the discussion. The number of initial concessions remains the same or increases in cases where the antagonist chooses to accept various readings of one and the same concession.

$\mathrm{C} 1$ : If the antagonist disambiguates her initial concessions by presenting $\operatorname{SD} f\left(C_{i} ; \alpha ; \alpha_{1}, \ldots, \alpha_{n}\right)$ or $\operatorname{FD} f\left(C_{i} ; \alpha ; \alpha_{1}, \ldots, \alpha_{n}\right)$ at stage $i$, then $C_{i+1}=f\left(C_{i} ; \alpha ; \alpha_{1}, \ldots, \alpha_{n}\right)$, that is, the result of disambiguating $C_{i}$ in this particular way.

The commitment store of the protagonist at stage one is empty. Given that the first stage of a dialogue contains the utterance of $\operatorname{ST} \varphi$ by the protagonist, $G_{2}$ only contains $\varphi$. The commitment store of the protagonist remains a singleton, but its element can become both increasingly disambiguated, if the protagonist chooses to disambiguate in the course of the dialogue, as well as increasingly complex, if the protagonist chooses to offer reasons for his standpoint or for premises that support his standpoint. As every argument put forward by the protagonist is an argument in favour of an element that is already part of his global argument at that stage, the protagonist is building a single, ever more complex defence of his position. This makes it convenient to determine that the content of $G_{i}$ is the tree-shaped structure of standpoint, reasons, connection premises and their relations, which represents the protagonist's defence at stage $i$. Thus, a first local argument, SsoT, would lead to the following element in the protagonist's commitment store:

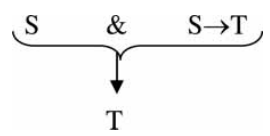

C2: If the protagonist utters $\operatorname{ST} \varphi$ at stage 1 , then $G_{2}$ only contains $\varphi$.

C3: If the protagonist utters $\psi \mathrm{SO} \varphi$ at stage $i$, then $G_{i+1}=$ contains $G_{i}$ with $\psi$ and $\psi \rightarrow \varphi$, connected with a horizontal line, written above the occurrence of $\varphi$ that was under attack and an arrow between the horizontal line and $\varphi$.

C4: If the protagonist at stage $i$ utters $\operatorname{SD} f\left(G_{i} ; \alpha ; \alpha_{1}, \ldots, \alpha_{n}\right)$ or $\operatorname{FD} f\left(G_{i} ; \alpha ; \alpha_{1}, \ldots, \alpha_{n}\right)$, then $G_{i+1}$ contains exactly $f\left(G_{i} ; \alpha ; \alpha_{1}, \ldots, \alpha_{n}\right)$, that is, the global argument such that each occurrence of $\alpha$ has been replaced with a disambiguating reformulation from the list $\alpha_{1}, \ldots, \alpha_{n}$, as indicated in the locution.

C5: If the protagonist at stage $i$ utters $\mathrm{WIG}_{j} ; \alpha, j<i$, then $\mathrm{GA}_{i+1}=\mathrm{GA}_{j}$. This enables the protagonist to withdraw his disambiguation, if it has proven to be linguistically inadmissible or interactionally irrelevant, by returning to an earlier version of his global argument. 
C6: If the antagonist at stage $i$ utters $\mathrm{WIC}_{j} ; \alpha, j<i$, then $\mathrm{CA}_{i+1}=\mathrm{CA}_{j}$. This enables the antagonist to renounce her disambiguation, if it has proven to be linguistically inadmissible or interactionally irrelevant, by returning to an earlier version of her store of concessions.

Part of each local argument in the global argument at some stage is a connection premise. The argument's connection premise is a conditional sentence having the argument's conclusion as its consequent and the conjunction of all reasons of that argument as its antecedent. Thus, if the protagonist has put forward the argument

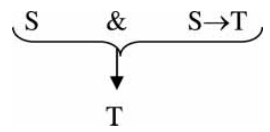

and subsequently disambiguates the distinct occurrences of S differently - for example by replacing S's first occurrence with $S_{1}$ and $S$ 's second occurrence with $S_{2}-$ a new connection premise is installed as part of the global argument at the next stage in the following way (see the first set of examples in Section 5.7):

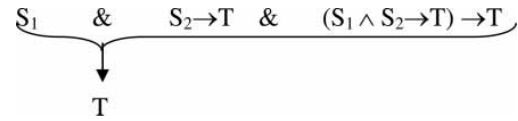

\subsection{The regulative dialogue rules}

There is one regulative rule.

R1: Do not use expressions that are actively ambiguous within the context of the discussion.

This rule can be violated in a dialogue in which none of the constitutive dialogue rules of ambiguity dialectic have been violated. Nevertheless, the constitutive dialogue rules incite the parties to obey the regulative rule or attempt to obey it as much as possible.

\subsection{The constitutive dialogue rules}

If an ambiguity correction or ambiguity criticism focusing on an expression $\alpha$ is not appropriate, for the reason that $\alpha$ is not really actively ambiguous, the disambiguation must be seen as unnecessarily nitpicking and opportunistic or as an attempt at filibustering. Therefore, when participant P1 is confronted with an ambiguity correction or an ambiguity criticism focused on $\alpha$ and mentioning $\alpha_{1}, \ldots, \alpha_{n}$ as the disambiguating reformulations, P1 needs to be able to test the linguistic admissibility of $\alpha_{1}, \ldots, \alpha_{n}$ as expressing possible senses of $\alpha$ and to test the interactional relevance of this multiplicity in meaning.

The dialogue model has the option to follow a procedure which determines whether the parties share linguistic norms that exclude one or more of the proposed disambiguating reformulations $\alpha_{1}, \ldots, \alpha_{n}$ as expressing something that $\alpha$ expresses in the context of use. I do not propose any details of such a procedure. The important thing here is that discussants can be seen as agreeing on a set of linguistic norms, and if they are able to further agree on the fact that a disambiguating reformulation $\alpha_{j}$ is linguistically inadmissible, then the ambiguity correction or ambiguity criticism counts as improper. If the result of the linguistic procedure is clearly positive or if the matter remains contentious or doubtful from a linguistic point of view, the disambiguation counts as having been sustained. Consequently, in cases of doubt, the presumption is on the side of 
the party who strives for more precision. How a relevance criticism is to be responded to depends on who is the target. The protagonist must show that there is a pseudo-disagreement, the antagonist a pseudo-agreement.

For most rules, it is stated in parentheses regarding what rule to apply if certain options are chosen.

D1: The protagonist starts the dialogue at stage 1 by uttering $\operatorname{ST} \varphi(\mathrm{D} 3)$.

D2: The parties move alternately.

D3: If stage $i$ contains the protagonist's standpoint $\operatorname{ST} \varphi$, then stage $i+1$ contains the antagonist's:

(a) $\mathrm{WH} \varphi(\mathrm{D} 4)$, or

(b) $\operatorname{SD} f\left(C_{i} ; \alpha ; \alpha_{1}, \ldots, \alpha_{n}\right)$ if $\alpha$ occurs in $\varphi$ and in $C_{i}$ (D6), or

(c) $\alpha A A \alpha_{1}, \ldots, \alpha_{n}$ if $\alpha$ occurs in $\varphi$ but not in $C_{i}$ (D6), or

(d) GIVE UP.

D4: If stage $i$ contains $\varphi$ as a standpoint, a reason or a connection premise and if stage $i+1$ contains the antagonist's challenge $\mathrm{WH} \varphi$, then stage $i+2$ contains the protagonist's:

(a) $\psi \mathrm{SO} \varphi(\mathrm{D} 5)$, or

(b) $\operatorname{SD} f\left(G_{i} ; \alpha ; \alpha_{1}, \ldots, \alpha_{n}\right)$ if $\alpha$ occurs in $\varphi$ but not in $C_{i}$ (D7), or

(c) $\alpha \mathrm{AA} \alpha_{1}, \ldots, \alpha_{n}$ if $\alpha$ occurs in $\varphi$ and in $C_{i}$ (D8), or

(d) GIVE UP.

D5: If stage $i$ contains $\chi_{1}, \chi_{2}, \ldots$ as unsupported elements (standpoint, reason or connection premise) of the protagonist's argument $\psi \mathrm{SO} \varphi$, or of the forced disambiguation $\operatorname{FD} f\left(G_{i}\right.$; $\left.\alpha ; \alpha_{1}, \ldots, \alpha_{n}\right)$, or of the ambiguity withdrawal $\mathrm{WIG}_{j} ; \alpha$ presented at $i$, or of the presented global argument $G_{i}$ presented at $i$, then stage $i+1$ contains the antagonist's:

(a) $\mathrm{WH} \chi_{j}$ for some $\chi_{j}$ (D4 or D10), or

(b) $\operatorname{SD} f\left(C_{i} ; \alpha ; \alpha_{1}, \ldots, \alpha_{n}\right)$ if $\alpha$ occurs in some $\chi_{j}$ and in $C_{i}$ (D6), or

(c) $\alpha \mathrm{AA} \alpha_{1}, \ldots, \alpha_{n}$ if $\alpha$ occurs in some $\chi_{j}$ but not in $C_{i}$ (D6), or

(d) GIVE UP.

D6: If stage $i$ contains the antagonist's spontaneous disambiguation $\operatorname{SD} f\left(C_{i} ; \alpha ; \alpha_{1}, \ldots, \alpha_{n}\right)$ or the antagonist's ambiguity criticism $\alpha \mathrm{AA} \alpha_{1}, \ldots, \alpha_{n}$, then stage $i+1$ contains the protagonist's:

(a) linguistic criticism LI? (D11 or D12), or

(b) relevance criticism RE? (D13), or

(d) disambiguation $\mathrm{FD} g\left(G_{i} ; \alpha ; \alpha_{1}, \ldots, \alpha_{n}\right)(\mathrm{D} 5)$.

D7: If stage $i$ contains the antagonist's $\mathrm{WH} \varphi[\alpha]$ and stage $i+1$ contains the protagonist's spontaneous disambiguation $\operatorname{SD} f\left(G_{i+1} ; \alpha ; \alpha_{1}, \ldots, \alpha_{n}\right)$, with $\varphi\left[\alpha_{j}\right]$ as part of the result, then stage $i+2$ contains the antagonist's:

(a) linguistic criticism LI? (D16), or

(b) $\mathrm{WH} \varphi\left[\alpha_{j}\right]$ (which functions here as a sustained relevance criticism) (D18), or

(d) $\mathrm{WH} \psi$ such that $\psi$ is a (yet) unchallenged reason or connection premise in $f\left(G_{i+1} ; \alpha\right.$; $\left.\alpha_{1}, \ldots, \alpha_{n}\right)$, joined by $\operatorname{FD} g\left(C_{i} ; \alpha ; \alpha_{1}, \ldots, \alpha_{n}\right)$, with which the ambiguity correction is acknowledged (D4).

D8: If stage $i$ contains the protagonist's ambiguity criticism $\alpha \mathrm{AA} \alpha_{1}, \ldots, \alpha_{n}$, then stage $i+1$ contains the antagonist's:

(a) linguistic criticism LI? (D17), or

(b) relevance criticism RE? (D19), or

(c) disambiguation $\operatorname{FD} g\left(C_{i} ; \alpha ; \alpha_{1}, \ldots, \alpha_{n}\right)(\mathrm{D} 9)$.

D9: If stage $i$ only contains the antagonist's forced disambiguation $\operatorname{FD} g\left(C_{i} ; \alpha ; \alpha_{1}, \ldots, \alpha_{n}\right)$, then $i+1$ contains the protagonist's forced disambiguation $\operatorname{FD} g\left(G_{i} ; \alpha ; \alpha_{1}, \ldots, \alpha_{n}\right)$ (D5). 
D10: If stage $i$ contains the antagonist's $\mathrm{WH} \varphi$, stage $i+1$ the protagonist's ambiguity criticism $\alpha \mathrm{AA} \alpha_{1}, \ldots, \alpha_{n}$, stage $i+2$ the antagonist's RE?, stage $i+3$ the protagonist's $\operatorname{FD} f\left(G_{i} ; \alpha\right.$; $\left.\alpha_{1}, \ldots, \alpha_{n}\right)$, with $\varphi\left[\alpha_{i}\right]$ as part of it and stage $i+4$ the antagonist's relevance criticism $\mathrm{WH} \varphi\left[\alpha_{i}\right]$, then stage $i+5$ contains the protagonist's $\mathrm{WIG}_{i} ; \alpha$ (D5).

D11: If stage $i$ contains the antagonist's $\operatorname{SD} f\left(C_{i} ; \alpha ; \alpha_{1}, \ldots, \alpha_{n}\right)$ and stage $i+1$ the protagonist's linguistic criticism LI?, then stage $i+2$ contains the antagonist's $\operatorname{FD} f\left(C_{i} ; \alpha ; \alpha_{1}, \ldots, \alpha_{n}\right)$ if the linguistic test is not clearly negative (D9) and $\mathrm{WIC}_{i} ; \alpha$ if the test is clearly negative (D15).

D12: If stage $i$ contains the antagonist's $\alpha \mathrm{AA} \alpha_{1}, \ldots, \alpha_{n}$ and stage $i+1$ the protagonist's linguistic criticism LI?, then stage $i+2$ contains the antagonist's $\alpha \mathrm{AA} \alpha_{1}, \ldots, \alpha_{n}$ if the linguistic test is not clearly negative (D6) and WI $\alpha$ if the test is clearly negative (D15).

D13: If stage $i$ contains the antagonist's $\operatorname{SD} f\left(C_{i} ; \alpha ; \alpha_{1}, \ldots, \alpha_{n}\right)$ or $\alpha \mathrm{AA} \alpha_{1}, \ldots, \alpha_{n}$ and stage $i+1$ the protagonist's relevance criticism RE?, then stage $i+2$ contains the antagonist's:

(a) $\mathrm{CO} \varphi\left[\alpha_{i}\right] ; \mathrm{WH} \varphi\left[\alpha_{j}\right](\mathrm{D} 14)$, or

(b) $\mathrm{WIC}_{i} ; \alpha$, when stage $i$ contains $\operatorname{SD} f\left(C_{i} ; \alpha ; \alpha_{1}, \ldots, \alpha_{n}\right)$ and $\mathrm{WI} \alpha$ when stage $i$ contains $\alpha \mathrm{AA} \alpha_{1}, \ldots, \alpha_{n}$ (D15).

D14: If stage $i$ contains the antagonist's relevance defence $\operatorname{CO} \varphi\left[\alpha_{i}\right] ; \mathrm{WH} \varphi\left[\alpha_{j}\right]$ ?, then stage $i+1$ contains the protagonist's:

(a) $\operatorname{FD} f\left(G_{i} ; \alpha ; \alpha_{1}, \ldots, \alpha_{n}\right)(\mathrm{D} 5)$, or

(b) GIVE UP.

D15: If stage $i$ contains the antagonist's ambiguity withdrawal $\mathrm{WI} \alpha$ or $\mathrm{WIC}_{i} ; \alpha$, then stage $i+1$ contains the protagonist's $G_{i}$ (D5).

D16: If stage $i$ contains the protagonist's $\operatorname{SD} f\left(G_{i} ; \alpha ; \alpha_{1}, \ldots, \alpha_{n}\right)$, and stage $i+1$ the antagonist's linguistic criticism LI?, then stage $i+2$ contains the protagonist's $\operatorname{FD} f\left(G_{i} ; \alpha ; \alpha_{1}, \ldots, \alpha_{n}\right)$ (D5) if the linguistic test is not clearly negative and $\mathrm{WIG}_{i} ; \alpha$ (the global argument before it was disambiguated) if clearly negative (D5).

D17: If stage $i$ contains the protagonist's $\alpha \mathrm{AA} \alpha_{1}, \ldots, \alpha_{n}$, and stage $i+1$ the antagonist's linguistic criticism LI?, then stage $i+2$ contains the protagonist's $\alpha \mathrm{AA} \alpha_{1}, \ldots, \alpha_{n}$ if the linguistic test is not clearly negative (D8) and $\mathrm{WIG}_{i} ; \alpha$ if clearly negative (D5).

D18: If stage $i$ contains the antagonist's $\mathrm{WH} \varphi$, stage $i+1$ the protagonist's $\operatorname{SD} f\left(G_{i+1} ; \alpha\right.$; $\left.\alpha_{1}, \ldots, \alpha_{n}\right)$ containing $\varphi\left[\alpha_{j}\right]$ as a disambiguation of the challenged $\varphi$, and stage $i+2$ the antagonist's relevance criticism $\mathrm{WH} \varphi\left[\alpha_{j}\right]$, then stage $i+3$ contains the protagonist's $\mathrm{WIG}_{i}$; $\alpha$ (D5).

D19: If stage $i$ contains the protagonist's ambiguity criticism $\alpha \mathrm{AA} \alpha_{1}, \ldots, \alpha_{n}$, and stage $i+1$ the antagonist's relevance criticism RE?, then stage $i+3$ contains $\operatorname{FD} f\left(G_{i+1} ; \alpha ; \alpha_{1}, \ldots, \alpha_{n}\right)$ (D5).

D20: The antagonist is not allowed to challenge $\varphi$ (standpoint, reason or connection premise) at stage $i$ if $\varphi$ is an element of $C_{i}$.

D21: At stage $k$ the use of expression $\alpha$ is not permittted if at an earlier stage $i$ there is an occurrence of $\alpha \mathrm{AA} \alpha_{1}, \ldots, \alpha_{n}$ or of $\operatorname{SD} f\left(C_{i} ; \alpha ; \alpha_{1}, \ldots, \alpha_{n}\right)$ or of $\operatorname{SD} f\left(G_{i} ; \alpha ; \alpha_{1}, \ldots, \alpha_{n}\right)$, unless there is a stage $j, i<j<l$, that contains either $\mathrm{WIG}_{j} ; \alpha$ or $\mathrm{WIC}_{j} ; \alpha$ or $\mathrm{WI} \alpha$.

D22: It is not permitted to raise exactly the same ambiguity criticism, linguistic criticism or relevance criticism.

\subsection{Win-and-loss rules}

There is one win-and-loss rule:

W1: The participant who utters GIVE UP loses the discussion and the other participant wins it. 


\subsection{Sample ambiguity dialogues}

In this section, I will illustrate the dialogue system using some examples. A branching of the sequence of moves stands for alternative courses of the ambiguity dialogue. $\mathrm{P}$ is short for Protagonist, A for Antagonist. The code refers to a rule that enables the move. The term 'fine' is short for 'W.B. must be fined'; 'drive' for 'W.B. is driving a car'; 'drive ${ }_{1}$ ' for 'W.B. is driving a car, in the broad sense of "driving"'; 'drive ${ }_{2}$ ' for 'W.B. is driving a car, in the strict sense of "driving".

(1) In the first set of examples, the antagonist raises an ambiguity criticism and the protagonist acknowledges it by disambiguating the global argument in somewhat different ways in the two dialogues.

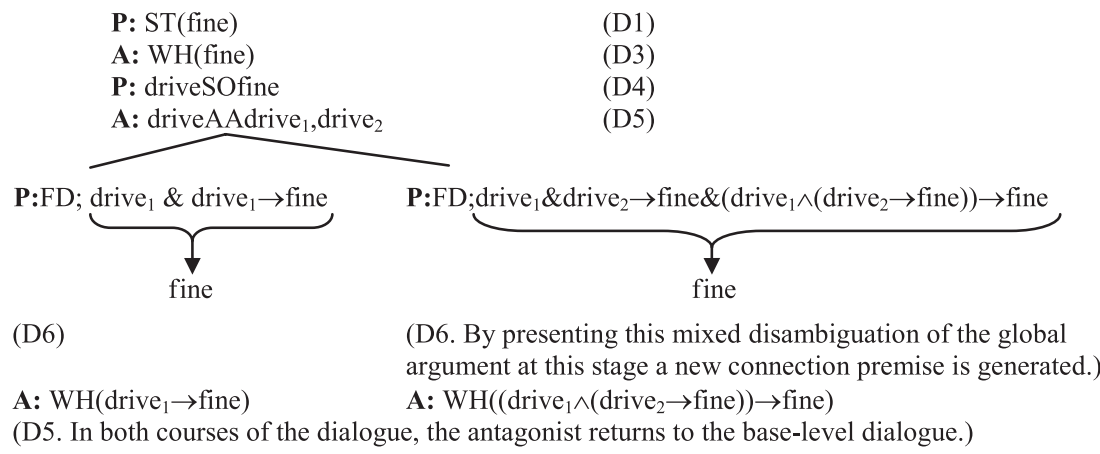

(2) In the second set of examples, the protagonist, out of fear of a pseudo-disagreement, spontaneously disambiguates his global argument and the antagonist responds critically by challenging the linguistic admissibility. In the branch on the left, the linguistic test does not prove negative, in the branch on the right, it does.

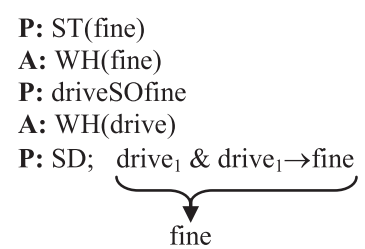

A: LI?

(D7. The antagonist challenges the linguistic analysis used in the spontaneous disambiguation.)

(D1)

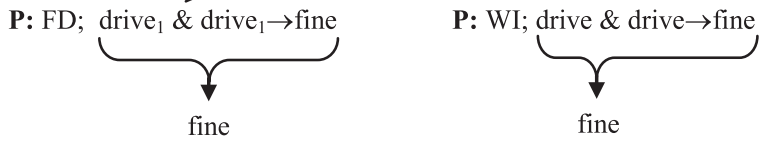

(D16. The left branch shows the protagonist's move when the linguistic test is not clearly negative. Here, the disambiguation is 'forced' and so the antagonist must return to the ground level dialogue by challenging an element of this new global argument. The right branch shows how the protagonist withdraws her disambiguated global argument and replaces it with the older global argument that does not employ the illicit disambiguation.) 
(3) In the third set of examples, the antagonist challenges the relevance of a spontaneous disambiguation offered by the protagonist.

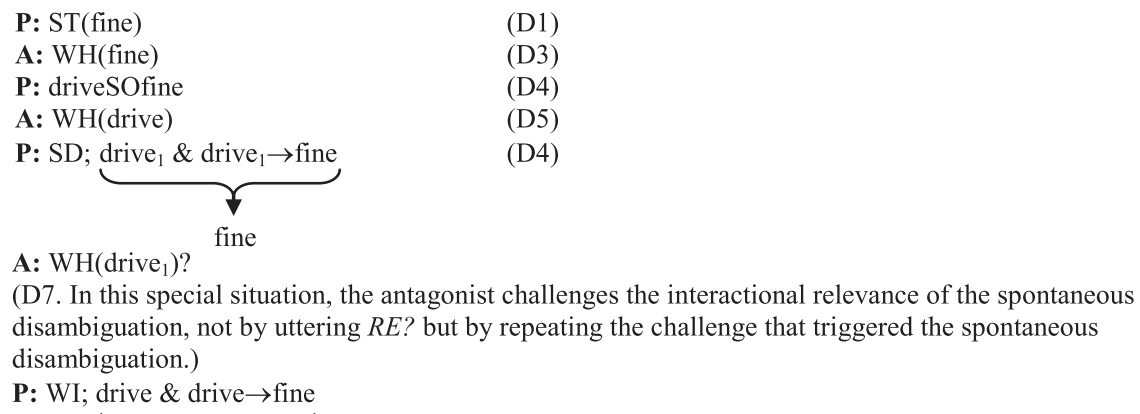

(D18. Given that the fourth stage does not, after all, seem to have produced a pseudo-disagreement, the disambiguation appears to be interactionally irrelevant and the protagonist is forced to withdraw the disambiguation and return to the base level dialogue by phrasing the global argument without the allegedly nitpicking reformulations.)

(4) In the fourth set of examples, the antagonist is herself partly responsible for the existence of the expression 'fine'. Consequently, the antagonist spontaneously disambiguates her set of initial concessions.
P: ST(fine)
A: WH(fine)
P: driveSOfine

A: $\mathrm{SD}$; drive; $\left\{\right.$ drive $_{1}$, drive $_{2} \rightarrow$ fine $\}$

(D5. The antagonist considers the possibility of becoming a victim of either a pseudo-agreement or a fallacy of equivocation. By spontaneously disambiguating the initial concessions, an attempt to avoid this outcome is made. It is assumed here that the antagonist's set of concessions only contained drive and drive $\rightarrow$ fine.)

P: RE?

A: $\mathrm{CO}\left(\right.$ drive $\left._{1}\right) ; \mathrm{WH}\left(\right.$ drive $\left._{2}\right)$

(D13. With this move the antagonist shows that a pseudo-agreement had been threatening the dialogue, for there is a willingness to concede the reason offered by the protagonist presented in the third stage in the one sense, but she would challenge the reason if disambiguated in the other direction. Note that within the system this move merely proves the relevance of the antagonist's spontaneous disambiguation of the concessions set. The protagonist does not (yet) need to start supporting drive ${ }_{2}$, having not chosen to disambiguate the argument in that way (yet).)

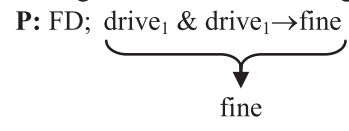

(D14)

A: $\mathrm{WH}\left(\right.$ drive $_{1} \rightarrow$ fine $)$

(D5. The antagonist is still in a position to maintain a critical stance, by challenging the connection premise.)

\section{Conclusion}

In this paper, an explication of the pejorative sense of 'ambiguous', called 'actively ambiguous', has been presented. It has been argued that even a normative model of reasonable and critical discussion ought not to ban active ambiguities altogether. Instead, it was proposed that the norms 
of persuasion dialogue must first enable each participant to put forward ambiguity criticisms as well as self-critical ambiguity corrections to induce them to improve their language, and second enable them to determine whether the ambiguity corrections or ambiguity criticisms are appropriate, thereby preventing nitpicking and filibustering.

A dialogue system, ambiguity dialectic, has been specified that satisfies six philosophical requirements on normative models of argumentative discussion that accommodate active ambiguities. Ambiguity dialectic is immanently dialectical, it strikes a balance between normative bite and a tolerance of imperfections, it implements the correct norms regarding the use of actively ambiguous expressions, it is organised in accordance with a dialectical division of labour, it strikes a balance between enabling meta-remarks and keeping to the topic at hand, and it starts from a clear conception of disambiguation. The model can be used to analyse and evaluate ways in which discussants deal with ambiguities, as well as for the further development of interaction protocols for agents attempting to achieve their argumentative aims in a reasonable way while using an imperfect language.

\section{Acknowledgements}

An earlier version of this paper was presented at the 'Dimensions of Ambiguity' conference at the Eberhard Karls University Tübingen, Tübingen, November 2009. I am very grateful for the comments made by the anonymous reviewers of this journal.

\section{Notes}

1. The approach that I adopt coheres with that of pragma-dialectics, as defended by van Eemeren and Grootendorst (1992, 2004).

2. The fourth component is part of the incomplete sketch of ambiguity dialogues in van Laar (2001).

3. If a protagonist defends a standpoint as true, rather than as merely acceptable to the antagonist, this may be considered as a special case when the thesis is defended ex concessis and when the concessions used are presented as true rather than merely accepted by the antagonist.

4. I follow Walton and Krabbe (1995) in characterising dialogue types by referring to the initial situation, the individual aims of the participants and the common goal of the dialogue. Ambiguity dialectic is a subtype of what Walton and Krabbe call permissive persuasion dialogue. If a dialogue participant abandons the aim of persuading an interlocutor, or resolving the difference of opinion, he or she is no longer involved in the kind of dialogue that fits the description of persuasion dialogue. However, in such a situation, we may nevertheless apply the norms of a persuasion dialogue in order to reconstruct and evaluate a contribution from an argumentative stance.

5. Krabbe and van Laar (2007) propose a typology of functions of reasoning.

6. These latter two pretences closely correspond to the objective of dialectical reasonableness and the objective of rhetorical effectiveness that constitute strategic manoeuvring, as examined by van Eemeren and Houtlosser (1999, 2002, 2005).

7. This is unproblematic if it is the protagonist who introduces the expression. In cases where the antagonist has used an expression in her initial concessions which, when used by the protagonist, turns out to be actively ambiguous, the antagonist remains partly responsible for any resulting problems, even though the protagonist is probably also in part to blame. In order to obtain a clear division of tasks ambiguity dialectic assigns in those cases the burden of solving these problems to the antagonist alone. See, for example, the fourth set of examples in Section 5.7.

8. Elsewhere (van Laar 2003) I have defended the notion that whether or not an argument constitutes a fallacy of equivocation is in part dependent upon what the addressee is willing to accept.

9. The immanent dialogical nature of the theory is in line with Hamblin's (1970) proposal to study ambiguity within the setting of a theory of charges. His idea is that given that we lack objective criteria for determining expressions that give rise to equivocation, we should study charges of equivocation. 
10. In the driving example, I assumed the parties to be engaged in a persuasion dialogue, thus not appealing to a judge with the power to decide who is right or wrong. (As a matter of fact, the judge did, in the end, settle the dispute by choosing the broader meaning of 'to drive' as the proper legal meaning.)

11. The theories of Mackenzie (1988, 1990), Walton (1996) and van Eemeren and Grootendorst (2004) do not provide participants with fully explicit linguistic devices for testing the correctness of ambiguity charges or the appropriateness of ambiguity corrections.

12. Different from, for example, Frege, Russell and Wittgenstein, who were striving after a logically ideal language, implying among other things an isomophism between syntactic expressions and semantic meanings, in this paper I picture dialogue participants who are trying to improve their language, if they think that is needed, without such high aspirations (cf. Soames 2003; Sullivan 2003).

13. This is not the case in Mackenzie $(1988,1990)$, where the critic who charges the arguer with equivocation chooses the disambiguation of the arguer's commitments.

14. Ambiguity dialectic specifies a procedure that enables the participants to, what Cohen (1966) phrased as, adapting meanings to truth-values. Cohen considers meanings as changeable. In case we are confronted with vague expressions, we are not forced to consider some of the sentences that contain them as either both true and false or neither true nor false. Instead, we may choose to adapt their meaning by 'precisification', in order to make the statement simply true or false. 'A special logic of vagueness may well be interesting, but it is not indispensible' (p. 293).

15. Because a participant in an ambiguity dialogue is allowed to request further disambiguations, again and again, there is no guarantee that the initial difference of opinion will be resolved by the parties. This consequence is shared by other models of reasonable discussion (Walton and Krabbe 1995; van Eemeren and Grootendorst 2004).

16. Ambiguity dialectic does not accommodate the option to retrace one's steps (Barth and Krabbe 1982, p. 76; cf. backtracking in Prakken 2005, p. 1016), by introducing an alternative response to a previous move from the interlocutor. As far as disambiguation is concerned, it would be interesting to extend the system so that a participant is enabled to retrace his steps and to try an alternative disambiguation.

17. Elsewhere, Walton proposes a dialogue system that starts from a clear division of labour (Walton 2007). However, this system is designed to clarify unclear expressions and is not specifically tailored to ambiguity problems.

18. Presenting a disambiguation is related to presenting a definition in the following way. If a participant disambiguates his or her position with respect to expression $\alpha$, he or she provides a kind of stipulative definition of $\alpha$ in that it is made clear how in this context this expression is to be understood. Moreover, it is also made clear for at least one other definition of $\alpha$ that this is not to be understood as providing $\alpha$ 's meaning. No method of stipulative definition need be excluded for the purpose of definition (Robinson 1972/1954). Disambiguation implies a definition, but not vice versa, for the reason that definitions can also be used to clarify the meaning of an expression which is not considered ambiguous. Presenting a disambiguation is less closely connected to dissociation, for a participant who disambiguates his or her position can, but need not claim that the disambiguation choice corresponds to the 'real meaning' of the ambiguous term (Perelman and Olbrechts-Tyteca 1969).

\section{References}

Barth, E.M., and Krabbe, E.C.W. (1982), From Axiom to Dialogue: A Philosophical Study of Logics and Argumentation, Berlin, New York: de Gruyter.

Cohen, L.J. (1966), The Diversity of Meaning, London: Methuen.

van Eemeren, F.H., and Grootendorst, R. (1992), Argumentation, Communication and Fallacies, Hillsdale, NJ: Lawrence Erlbaum.

van Eemeren, F.H., and Grootendorst, R. (2004), A Systematic Theory of Argumentation. The PragmaDialectical Approach, Cambridge: University Press.

van Eemeren, F.H., and Houtlosser, P. (1999), 'Delivering the Goods in Critical Discussion', in Proceedings of the Fourth International Conference of the International Society for the Study of Argumentation, eds. F.H. van Eemeren, R. Grootendorst, J.A. Blair, and C.A. Willard, Amsterdam: Sic Sat, pp. 168-167. 
van Eemeren, F.H., and Houtlosser, P. (2002), 'Strategic Manoeuvring in Argumentative Discourse: A Delicate Balance', in Dialectic and Rhetoric: The Warp and Woof of Argumentation Analysis, eds. F.H. van Eemeren and P. Houtlosser, Dordrecht: Kluwer, pp. 131-159.

van Eemeren, F.H., and Houtlosser, P. (2005), 'Theoretical Construction and Argumentative Reality: An Analytical Model of Critical Discussion and Conventionalised Types of Argumentative Activity', in The Uses of Argument: Proceedings of a Conference at McMaster University, ed. D. Hitchcock, Hamilton: Ontario Society for the Study of Argumentation, pp. 75-84.

Hamblin, C.L. (1970), Fallacies, Virginia: Vale Press.

Ide, N., and Veronis, J. (1998), 'Introduction to the Special Issue on Word Sense Disambiguation: The State of the Art', Computational Linguistics, 24, 1-40.

Keefe, R. (2000), Theories of Vagueness, Cambridge: Cambridge University Press.

Krabbe, E.C.W. (1997), Wat is Eigenlijk een Drogreden?, inaugural speech at the University of Groningen, Groningen: E.C.W. Krabbe.

Krabbe, E.C.W. (2001), 'Strategies in Dialectic and Rhetoric', in Argumentation and its Applications, eds. V. Hansen, C.W. Tindale, J.A. Blair, R.H. Johnson, and R.C. Pinto, CD-ROM, ISBN 0-9683461-2-X, Proceedings from the Conference of the Ontario Society for the Study of Argumentation, 17-19 May, 2001, University of Windsor, Windsor, Ontario: University of Windsor.

Krabbe, E.C.W. (2002), 'Profiles of Dialogue as a Dialectical Tool', in Advances in Pragma-Dialectics, ed. F.H. van Eemeren, Amsterdam: Sic Sat; Newport News, VA: Vale Press, pp. 153-167.

Krabbe, E.C.W. (2003), 'Metadialogues', in Anyone Who has a View: Theoretical Contributions to the Study of Argumentation, eds. F.H. van Eemeren, J.A. Blair, Ch.A. Willard, and A.F. Snoeck Henkemans, Dordrecht: Kluwer, pp. 83-90.

Krabbe, E.C.W., and van Laar, J.A. (2007), 'About Old and New Dialectic: Dialogues, Fallacies, and Strategies', Informal Logic, 154, 27-58.

van Laar, J.A. (2001), 'Ambiguity in a Dialectical Perspective', Informal Logic, 21, 245-266.

van Laar, J.A. (2003), 'The Dialectic of Ambiguity: A Contribution to the Study of Argumentation', PhD dissertation, University of Groningen, Groningen. http://irs.ub.rug.nl/ppn/249337959

Lewis, D. (1982), 'Logic for Equivocators', Nous, 16, 431-441.

Mackenzie, J. (1988), 'Distinguo: The Response to Equivocation', Argumentation, 2, 465-482.

Mackenzie, J. (1990), 'Four Dialogue Systems', Studia Logica, 49, 567-583.

McBurney, P., and Parsons, S. (2002), 'Games That Agents Play: A Formal Framework for Dialogues between Autonomous Agents', Journal of Logic, Language and Information, 11, 315-334.

Naess, A. (1953), Interpretation and Preciseness: A Contribution to the Theory of Communication, Oslo: Dybwad.

Naess, A. (1966), Communication and Argument: Elements of Applied Semantics, Oslo: Universitetsforlaget; London: Allen and Unwin.

Navigli, R. (2009), 'Word Sense Disambiguation: A Survey', ACM Computing Surveys, 41, 10.1-10.69.

Parsons, S., Wooldridge, M., and Amgoud, L. (2003), 'Properties and Complexity of Some Formal Inter-agent Dialogues', Journal of Logic and Computation, 13, 347-376.

Perelman, Ch., and Olbrechts-Tyteca, L. (1969), The New Rhetoric: A Treatise on Argumentation, Notre Dame: University of Notre Dame Press.

Pinkal, M. (1995), Logic and Lexicon, Dordrecht: Kluwer.

Pollock, J.L. (1995), Cognitive Carpentry: A Blueprint for How to Build a Person, Cambridge, MA: MIT.

Prakken, H. (2005), 'Coherence and Flexibility in Dialogue Games for Argumentation', Journal of Logic and Computation, 15, 1009-1040.

Rescher, N. (1977), Dialectics: A Controversy-Oriented Approach to the Theory of Knowledge, Albany, NJ: State University of New York Press.

Robinson, R. (1972/1954), Definition, Oxford: Clarendon.

Soames, C. (2003), Philosophical Analysis in the Twentieth Century (Vol. 1), Princeton, NJ: Princeton University Press.

Sullivan, A. (2003). 'Introduction', in Logicism and the Philosophy of Language: Selections from Frege and Russell, ed. A. Sullivan, Peterborough: Broadview, pp. 15-90. 
Thoreau, H.D. (1963/1849), On the Duty of Civil Disobedience (With an introduction by Gene Sharp), London: Peace News.

Walton, D.N. (1996), Fallacies Arising from Ambiguity, Dordrecht: Kluwer.

Walton, D.N. (1998), The New Dialectic: Conversational Contexts of Argument, Toronto: University of Toronto Press.

Walton, D.N. (2007), 'The Speech Act of Clarification in a Dialogue Model', Studies in Communication Sciences, 7, 127-159.

Walton, D.N., and Krabbe, E.C.W. (1995), Commitment in Dialogue: Basic Concepts of Interpersonal Reasoning, New York: State University of New York Press.

Wells, S., and Reed, C. (2005), 'Formal Dialectic Specification', in Argumentation in Multi-Agent Systems (Proceedings of ArgMAS 2004), eds. I. Rahwan, P. Moraitis, and C. Reed, New York: Springer, pp. 31-43. 